\title{
Publisher's Note: Non-Fermi Liquid Behavior Close to a Quantum Critical Point in a Ferromagnetic State without Local Moments [Phys. Rev. X 5, 011026 (2015)]
}

E. Svanidze, L. Liu, B. Frandsen, B. D. White, T. Besara, T. Goko, T. Medina, T. J. S. Munsie, G. M. Luke, D. Zheng, C. Q. Jin, T. Siegrist, M. B. Maple, Y. J. Uemura, and E. Morosan

(Received 18 March 2015; published 26 March 2015)

DOI: 10.1103/PhysRevX.5.019902

Subject Areas: Condensed Matter Physics

This paper was published online on 4 March 2015 with an omission in the Acknowledgments. "T. B. and T. S. acknowledge support by the Department of Energy, Basic Energy Science, Division of Materials Science under award DE-SC0008832, and the National High Magnetic Field Laboratory, which is supported by National Science Foundation Cooperative Agreement No. DMR-1157490 and the State of Florida." has been added online as the last sentence as of 16 March 2015. 